\title{
Sanguineti, Don Chisciotte, il viaggio e alcune occasioni spagnole
}

\author{
Franco Vazzoler \\ Università di Genova \\ franco.vazzoler@lettere.unige.it
}

\begin{abstract}
Questo «omaggio» al poeta recentemente scomparso (uno dei maggiori intellettuali italiani del Novecento) prende spunto dalla pubblicazione in Varie ed eventuali (l'ultima raccolta poetica) dei Frammenti da "Invenzione di Don Chisciotte» (un testo del 1949), dove il personaggio cervantino è proposto come «viaggiatore sensibile». Si propone una lettura delle "poesie di viaggio" di Sanguineti ispirate a luoghi spagnoli e catalani. Partendo dalla poetica del "piccolo fatto vero», vi è sviluppata la dimensione-tema della «bilocazione» (come la chiamerà in Cose 63), che si rivela, nei poli dello sdoppiamento e dell'assenza, come uno dei nodi fondamentali che l'esperienza poetica del viaggio mette in evidenza.

Parole chiave: Sanguineti; Poesia; Viaggio; Sdoppiamento; Assenza.
\end{abstract}

\section{Abstract}

This «tribute» to the recently deceased poet (one of the leading Italian intellectuals of the 20 th century) is inspired by the publication of a piece from 1949, Frammenti da "Invenzione di Don Chisciotte» (Fragments from "The Invention of Don Quixote»), in Varie ed eventuali - his last poetry collection, where the Cervantes character is presented as a «sensitive traveller». The paper revisits Sanguineti's «travel poems», which were inspired by Spanish and Catalan places. Starting from the poetry of the "piccolo fatto vero" (small true event), this paper develops the dimension/topic of «bilocazione» — as he calls it in Cose 63- which is revealed, in terms of duality and absence, as one of the key topics highlighted by his poetic experience of travels.

Keywords: Sanguineti; Poetry; Travel; Duality; Absence.

Ad apertura di Varie ed eventuali, ${ }^{1}$ l'ultimo suo libro («un libro che si pubblica postumo, ma non è un libro postumo»), ${ }^{2}$ Edoardo Sanguineti ha collocato un testo che porta la data 1949: Frammenti da "Invenzione di Don Chisciotte», la cui singo-

1. Edoardo Sanguineti, Varie ed eventuali. Poesie 1995-2010, Milano: Feltrinelli, 2010, p. 7-9.

2. Niva LorenZInI, Postfazione, ibidem, p. 159. 
larità e importanza sono state giustamente e immediatamente messe in evidenza da una delle sue prime lettrici, Niva Lorenzini, nella Postfazione che lo correda.

Recuperati da Sanguineti nella loro veste originale, scritti a macchina su tre fogli, ed offerti a Mimmo Paladino nell'estate del 2006 mentre l'artista stava ultimando, con Corrado Bologna, il lungometraggio Quijote, questi Frammenti erano venuti a far parte del film, dove Sanguineti stesso li recitava. ${ }^{3}$ Erano poi apparsi nel 2006, prima in tiratura limitata in una plaquette accompagnati da una incisione di Mimmo Paladino e da un CD in cui erano letti da Toni Servillo, e successivamente, in forma di riproduzione fotografica del dattiloscritto, ${ }^{4}$ sul numero della rivista "Critica del testo», che aveva per titolo, I mondi possibili del Quijote, in occasione del 400 anniversario del capolavoro cervantino, preceduti da una introduzione di uno dei curatori della rivista, Corrado Bologna, ${ }^{5}$ che ricostruiva l'occasione della loro presentazione al museo Peggy Guggenheim di Venezia, avvenuta il 5 settembre di quell'anno, a corredo, a sua volta, della presentazione di una edizione del Quijote, curata da Bologna e illustrata dagli acquarelli di Mimmo Paladino, pubblicato da Editalia-Poligrafico dello Stato, mentre, quasi contemporaneamente, il lungometraggio Quijote di Paladino veniva presentato, nella sezione Orizzonti della Mostra del Cinema.

Della presentazione di Venezia, al museo Guggenheim il 5 settembre 2006, esiste un video in cui, oltre alla sua lettura di una parte della Invenzione, è registrato l'intervento di Sanguineti, da cui si ricava che il testo di Invenzione di Don Chisciotte, insieme ad un altro, Invenzione di Don Giovanni, faceva parte del progetto, poi abbandonato, di un libro che avrebbe dovuto avere il titolo di Composizioni. ${ }^{6}$

Al film di Paladino fa riferimento Niva Lorenzini, nel segnalare l'apparente "anomalia cronologica» ${ }^{7}$ della presenza di questo testo nell'ultima raccolta di Sanguineti, ${ }^{8}$ toccando il problema essenziale di questo «recupero», che ha

3. Come risulta dai crediti del lungometraggio, indicati sul sito della casa produttrice del film e scaricabili in pdf all'indirizzo <http://www.ananascinema.com/ita/crediti-ita.htlm>, Sanguineti vi figura nel ruolo di «Poeta».

4. Con alcune notazioni, secondo la testimonianza di Bologna, di Paladino, relative all'inserimento del testo nella sceneggiatura, addirittura numerando e citando le sequenze («schermo spalle», «camminata dx», "profilo (lago)», «nuvole»), il luogo della ripresa («disegno teatro india»), un attore («dopo Cucchi»: è Enzo Cucchi, nella parte del Mago Merlino).

5. Corrado Bologna, «La novissima Invenzione di Don Chisciotte di Edoardo Sanguineti», in Critica del testo, IX, 1-2, 2006, p. 385-396 (il testo di Sanguineti è alle p. 397-399). Mentre questo saggio era in bozze, Corrado Bologna è tornato su questi temi, con la relazione Fra Sancho e Don Chisciotte, in occasione del convegno per Edoardo Sanguineti: lavori in corso (Genova, 12-14 maggio 2011), dei cui atti è prevista la pubblicazione.

6. visibile sul sito <http://www.labiennale.tv> Cfr. anche: <http://www.editalia.it/>.

7. A proposito dell'origine di questo testo la Lorenzini afferma che all'epoca Sanguineti era stato suggestionato dal film di Pabst (Postfazione, cit., p. 160).

8. Lo considera addirittura come «nucleo costitutivo» del film e «motivo ispiratore» del Don Chisciotte «acquarellato» del pittore, probabilmente pensando le animazioni che accompagnavano nel Cd la voce recitante di Servillo. 
il valore soprattutto di una «riemersione». Se Bologna scrive che le parole di quel vecchio testo vi «galleggiano come schegge di un iceberg dissolto nel tempo [...] affiorano», Sanguineti ne parla come del «risuscitare un vecchio fantasma». Il materiale verbale, in effetti, vi viene rielaborato e trattato da Sanguineti come se fosse di un altro (dice Sanguineti, nel video: «l'ho sforbiciato perché non è più cosa mia») e poi rimontato da Paladino "come in un gioco di specchi perfettamente consono alle poetiche dei due artisti».?

Insomma, pensando alle date di questi recuperi, la riemersione di questo testo (2006) va a collocarsi quasi al centro cronologico-ideale di questo nuovo libro (i cui estremi, indicati dal titolo sono 1995-2010), da un lato dandogli il tono («dà il la a tutto il volume»: ancora Lorenzini), dall'altro come condensandolo. ${ }^{10} \mathrm{Ma}$ al tempo stesso, il riemergere del personaggio-tema di Chisciotte, di allora, in quest'ultima fase sanguinetiana, avviene dopo esser ri-comparso in Corollario 33 nell'immagine del «bronzeo ronzinante (in carta pesta)», quasi a far assonanza col «sono un bronzo di Riace insecchito, ingobbito (e un po' invecchito)» di Cataletto 1.

Il personaggio (o forse, ancor meglio, il progetto di personaggio) di Don Chisciotte 1949-2006 di Varie ed eventuali (2010), "homme plein de sens», senza uno spazio e un tempo precisato, ma posto all'inizio di quella che potrebbe essere definita come la «modernità» («io apro il tempo che viene, come una porta o una finestra: - o come una qualunque cosa chiusa:») sembra catalizzare, allora, un tema che dall'inizio alla fine ha percorso gran parte della poesia di Sanguineti, quello del viaggio: «viaggiatore sensibile», «io sono l'uomo che deve partire», «la tua partenza è soprattutto la mia».

Posto in uno spazio che non ha nessuna connotazione geografica («di fronte a tutti i luoghi della terra») può, allora, autodefinirsi «uomo delle nuvole», e colui che «nei paesaggi colloca figure», in una prima intuizione di quella identificazione fra «vivere» e «scrivere» che sarà determinante per tutto il futuro di Sanguineti e che qui sembra risentire di un'eco boccacciana in quell'appello alla «buona brigata». ${ }^{11}$

Va ricordato anche (lo fa Bologna nella presentazione citata precedentemente) che Paladino aveva realizzato anche un libro d'artista pubblicato dall'editore modenese Roberto Gatti, in 100 esemplari, contenente 6 incisioni all'acquaforte e acquatinta, collage, fustellatura, impressioni tipografiche, tecniche miste e accompagnato da un $\mathrm{Cd}$ in cui Mimmo Cuticchio aveva inciso il "cunto» Sui sentieri di Don Chisciotte, questa volta accompagnato da quattordici poesie di Giuseppe Conte.

9. Corrado Bologna, cit., p. 393.

10. A questi recuperi, d'altra parte, che rompono la regolare scansione cronologica delle raccolte fino a Segnalibro, Sanguineti ci aveva già abituati raccogliendo in Senzatitolo (1992) una serie di testi, che poi in Il gatto lupesco corrono paralleli ad un discorso inizialmente ancora ordinato cronologicamente (almeno fino a Rebus, e che solo Corollario e Cose sembrano parzialmente riprendere), come confermerà la struttura in due parti di Mikrokosmos che mantiene una stretta sequenza cronologica solo nella prima. Di questo modo (di questi modi) di costruire i libri (sia di poesia, quanto le raccolte di prose saggistiche e giornalistiche) bisognerà che primo o poi qualcuno si occupi a fondo.

11. «Don Chisciotte parla, state in silenzio ad ascoltare, / voi che siete una buona brigata / Don Chisciotte si espone e si dichiara e si spiega e si dimostra / —questo è Don Chisciotte» 
Ed è il primo comparire — prima della riemersione dalla palus putredinis di Laborintus - di quella figura di viaggiatore che poi, man mano, andrà a "collocare figure» sempre più precise in "paesaggi» sempre meglio definiti e registrati attraverso toponimi sempre più dettagliati nelle raccolte successive.

Se in Laborintus (1956) le indicazioni di toponimi (la Dead Valley, ${ }^{12}$ il ponte della luna, ${ }^{13}$ la Paradise Valley, ${ }^{14}$ il lago di Sompunt), ${ }^{15}$ quasi tutti contenenti un significato già nel nome, al punto da poter sembrare luoghi immaginari (l'Eldorado club, ${ }^{16}$ la sala da ballo El Flamingo), ${ }^{17}$ sono soprattutto luoghi simbolici, come le precise indicazioni lunari, o conservano un' "aura" artistico-letteraria, ${ }^{18}$ è a partire dalla piazza Chanoux (ad Aosta) — se non me ne sono sfuggiti di precedenti- che il toponimo (con registrazione della data, anche) presente nelle poesie, è legato ad una precisa occasione biografica, erotica ovviamente in questo caso (Erotopegna 1); in seguito le prime determinazioni di luoghi e date compariranno in Purgatorio dell'Inferno (1964), in concomitanza con l'allargarsi delle sue relazioni nazionali ed internazionali — di qui le poesie che assumono l'aspetto di appunti delle discussioni («verbali») ${ }^{19}$ e ricordi di collaborazioni ${ }^{20}$ _, ma anche di momenti di divertimento e d'avventura (come il paesaggio della Normandia, in 7), le visite ai musei, le strade di Parigi, ecc. É qui, infatti, che si fonda quel modo di poetare citando non solo i testi, ma anche le minime occasioni della biografia, registrate con puntigliosa pedanteria, che rimarrà poi la caratteristica di tanta poesia successiva. Per il momento è una geografia già definita, ma ancora abbastanza ristretta (l'Italia, la Francia), che nelle sette poesie di T.A.T., si estende anche al Belgio e agli Stati Uniti, ${ }^{21}$ ma è imminente il primo vero e proprio libro di viaggio, Reisebilder, che comprende 51 poesie del 1971 (scritte fra giugno e ottobre, fra

(p. 7). Molto più tardi, rispondendo al questionario di Erminio Risso (in Edoardo SANGUINETI, Mikrokosmos. Poesie 1951-2004, a cura di Erminio Risso, Milano: Feltrinelli, 2004, p. 326-328), se non includerà Don Chisciotte fra i «suoi eroi della finzione» (scegliendo Amleto, Faust, Woyzech), indicherà in Cervantes (accanto a Petronio e Stendhal) uno dei «suoi autori in prosa».

12. Laborintus 1 (in Edoardo Sanguineti, Segnalibro. Poesie 1951-1981, Milano: Feltrinelli, $2010^{1}$ [1982], p. 13).

13. «Der Mondbrücke», in una città della Svizzera (Laborintus 8, ibidem, p. 23).

14. Laborintus 10 , ibidem, p. 25.

15. Laborintus 23, ibidem, p. 43.

16. Laborintus 4, ibidem, p. 17.

17. Laborintus 7, ibidem, p. 21.

18. Come la proustiana Châteaudun o i luoghi di Van Gogh di Laborintus 19 (ibidem, p. 39). Per questi, come per gli altri, si cfr. Erminio Risso, Laborintus di Edoardo Sanguineti. Testo e commento, Lecce: Manni, 2006.

19. Penso ad esempio alle poesie 2 (novembre1960), 3 (marzo 1961), 17 (novembre 1963) per gli incontri di Cerisy e Palermo (cfr. Edoardo Sanguineti, Segnalibro. Poesie 1951-1981, cit., p. 72, 74, 90).

20. La poesia 6 (ibidem, p. 79), testimonianza importante della nascita di Passaggio.

21. Alamosa in Colorado è nominata con tanto di distanze in 3 (una delle poesie più interessanti da questo punto di vista). Allo stesso vanno riferite (hanno tutte la data di ottobre 1967) le poesie 3-6 (ibidem, p. 95-102). 
Olanda e Germania), cui seguiranno le 67 Postkarten dell'omonima raccolta scritte fra il 1972 e il 1977.

In queste raccolte, in cui si afferma ormai la poetica del «piccolo fatto vero", ${ }^{22}$ il viaggio diventa l'occasione privilegiata per imporre all' «io» del poeta quel carattere di «io per le strade» — secondo la felice formula di Elisabetta Baccarani ${ }^{23}$ - in cui l'originaria tensione al partire, che abbiamo vista in Don Chisciotte, si fa prassi di vita e si concretizza in itinerari reali. Ma non tutti i libri (o le singole sezioni) sono caratterizzati, come avveniva in Reisebilder, da un itinerario. Se, infatti, Mauritshuis (agosto 1986) è tutto concentrato nello spazio e nel tempo della visita alle sale del museo dell'Aja, le occasioni di viaggio (e le distanze delle varie mete) si moltiplicano, moltiplicandosi nella vita di Sanguineti, nelle raccolte successive che, come Mauritshuis, confluiranno successivamente e complessivamente in Il gatto lupesco. ${ }^{24}$

L'esorbitante geografia di Corollario (1977), che spazia dal Sud America, alla Palestina, al Nord Africa ai più abituali ambienti europei, pur sempre attenta agli orari degli aerei o dei treni, ai menu, dà comunque luogo a microsequenze, come quella del soggiorno a Barcellona (marzo 1996), che viene a costituirsi come la tappa di «una molle autoanalisi». Significativo, in questo senso, la numero 42, di fronte all' hanging model di Gaudì — probabilmente con in mano un opuscolo illustrativo, in spagnolo e in inglese- dove «dentro quel buio criptico», le "strutture più interiori» dell'io si presentano come «un folle enigma», come "una questione ipercomplessa» di fronte all'ambigua fascinazione prodotta dai «saquitos llenos de pernigones» della "maqueta invertida» nel «buio criptico» della basilica della Sagrada famiglia. ${ }^{25}$

Ma più spesso sono le esperienze erotiche (sotto forma di tradimento coniugale) a costituire un aspetto sempre presente in questi viaggi. Si veda ancora, sempre a Barcellona, nella n. 43 (che precede il commiato della n. 44) il «pistolone scarlatto / acceso, fondamentalmente bucato in punta, semieretto semirigido", la "fodera pelosa del mio picio» nel momento di un amplesso («per accoppiarci» (per «accopparci, si può persino sospettare), cuore a cuore, su sabbie desolate»); oppure l'addio all'aeroporto («amarti fu un dispendio, un lusso un vistoso sciupio»). ${ }^{26}$

L' erotismo aveva i caratteri dell'enigma e dell'ossessione nella contigua n. 41, la prima della serie barcellonese, incentrata sulla quête della statua "di un Eros, sbiancato e mutilato».

È in compagnia di altre tre persone (di una, una donna, cita anche il nome: Coral; poi ne evoca un'altra, Carmen), e la scena è il labirinto di siepi del Parc d'Horta (il giardino botanico di Barcellona):

22. Esplicitamente enunciata nella poesia 49 di Postkarten (ibidem, p. 209).

23. Elisabetta BACCARANI, «La persona migliore dello schermo. Modi ed evoluzioni dell'autobiografismo nella poesia di Edoardo Sanguineti», in Autobiografie in versi. Sei poeti allo specchio, a c. di M.A. BAZZOCCHI, Bologna: Pendragon, 2002, [p. 149-180], p. 167.

24. Edoardo Sanguineti, Il gatto lupesco. Poesie (1982-201), Milano: Feltrinelli, 2002, (le poesie di Mauritshuis sono alle p. 171-179).

25. Ibidem, p. 301.

26. Ibidem, p. 302. 
per arrivare a un Eros, sbiancato e mutilato (gli mancano due mani e, quasi, un braccio): (gli mancano anche tante frecce, per una glorificazione impietosa, impietosita, martirizzabile triste come sta), noi ci siamo perduti e riperduti (in quattro compresa una Coral), dentro el laberint: che lì all'ingresso si legge, dopo un entre, un saldras (cui tiene dietro, cortese, un sencillo):

(più tardi poi, da una Carmen, ebbi notizia scritta che, in qualche modo, in certo senso, donna gentile può dirsi xeitosa): (ma qui è un dedalo ovunque, uso disabili): ${ }^{27}$

É un eros grottescamente martirizzato («gli mancano due mani e quasi, / un braccio): (gli mancano anche tante frecce, per una glorificazione impietosa, / impietosita, martirizzabile come sta)»), a dar vita ad un gioco complesso di allusioni e mascheramenti metaforici (oltre alla statua mutilata, il labirinto), mentre gli inserti in spagnolo mimano un gioco linguistico («entre», «saldras», «sencillo») introdotto come se fosse un rebus ("dopo», "cui tiene dietro»).

E le prime sette poesie di Rebus sono scritte proprio in occasione di un viaggio in Spagna (Saragoza, Salamanca, Valencia, Santiago di Compostela, Toledo, Madrid), nel maggio $1984 .^{28}$

Nella prima, l'amosfera grottesco-espressionista è subito evocata dall'allusione al film di Sternberg con la Dietrich («[...] è stato un angelo (a Zaragoza è azul, dove è faticoso unratizzarsi sufficientemente):»), passando poi alla citazione di una canzone del gruppo rock-pop («rockeros») dei Golpes Bajos il cui titolo (Malos tiempos para la lírica) è immediatamente deformato con la traduzione italiana di un'espressione latina diventata proverbiale e inframmezzato $\mathrm{da}$ un commento che utilizza, anche in questo caso, un elemento popolare spagnolo e di Saragoza in particolare: «[...] che mali tempi corrono, deo gratias, e grazie alla Madonna / del Pilar, para la lírica, in genere»; sviluppando poi l'offerta proveniente da "para la lírica " (interpretata come «opera lirica»), per citare L'Incoronazione di Poppea, di Monteverdi nella deformazione caricaturale di un cabaret ("café cantante») di vecchia tradizione (la Plata e ne dà anche la localizzazione «nel Tubo», quartiere di Saragoza):

[...] (e dove il poetico si incarna in un urneronillo, che si disagrippinò al primo strillo, e che si spera, naturalmente, assassino d'assalto, un'altra volta un tanto, che è un pochino):

si vocalizzò, popputo e luminoso, gigantesco sciantoso, nell'archeologico retablo della Plata, nel Tubo, tra gli Hysterostudenti e prototuristici: $[\ldots]^{29}$

27. Ibidem, p. 300 .

28. Rebus è, inizialmente, la prima sezione di Bisdibis (Milano: Feltrinelli, 1987). I nomi delle città compaiono come titolo delle sette poesie pubblicate nel 1995 in Cuadernos de Filología italiana (n. 5, p. 327-331) con il titolo "Rebus 1984 (BISDIBIS)». Nello stesso numero compare una Entrevista a Edoardo Sanguineti, a cura di Rosario SCRIMERI e Aurora CONDE (p. 381-387).

29. Edoardo SANGUineti, Il gatto lupesco, cit., p. 41. 
Si delinea così quel percorso — segnalato dall" "unrattizzarsi» inizialeverso «un degrado o una degradazione» che riunisce «destinazione» e "destino».

Come sottolineerà più tardi l'autocommento, il progetto di un ulteriore sviluppo della poetica dell'impersonalità che è all'origine di tutto Rebus («Avevo in mente una poesia, che per così dire, desoggettivandosi a fondo, nascesse dalle cose stesse, quasi esentata da qualunque intervento autoriale»), ${ }^{30}$ è in realtà piuttosto una strategia autoanalitica, una autoterapia per cui è indispensabile guardare come da fuori il logorarsi dell' io.

L'esito può essere, allora, l'autoironia con cui osserva la propria goffaggine, che ricorda certi personaggi di romanzi beckettiani («mi inceppo, mi incespico, / sballottato sballato, sbalordito»), ${ }^{31}$ paragonandosi ad un «pelele» (uomo di paglia, spaventapasseri): "cadendo come cado e dove cado, saltandomi e storcendomi, sbando / di qua e di là» (Rebus3), o rappresentandosi come «l'ombra / di un pellegrino malinconico e alcolico [...] un dissoluto acuto, / soluto dentro i gorghi del torres» in un «fininsterresco paesino, paradisino / campesino» della Galizia (Rebus 4).

$\mathrm{E}$ ancora l'auto-ironia è risolta in giochi verbali ("(ti faccio appunto punto, smunto emunto: il tuo / confusionalmente compunto:)») nella conclusione della n. 6.

Questo autoironico osservarsi da fuori culmina nell' "esplosione di felice follia» per aver assistito «in prima fila, / e in prima persona, in proiezione» al proprio "pomposissimo entierro». 32

Qui il riferimento è senz'atro all' Entierro del conde de Orgaz, il dipinto di El Greco che si trova nella chiesa di Santo Tomé di Toledo: ennesima occasione di autorappresentazione della propria morte, nella spettacolarità di una sepoltura barocca, che come nota Weber, si riallaccia ai "toni testamentari» della conclusione del rebus precedente, ${ }^{33}$ tono testamentario che si affaccia anche qui nella devastante dimensione ossimorica con cui è osservata la propria vita: "fedeltà irriconoscibile», "costanza sfigurata», "tenacia guasta». E questo, dice, è «il mio segreto». Segreto rivelato in una raccolta che si chiama Rebus e che doveva essere il manifesto di una poesia impersonale, fatta, cioè, con le cose (secondo la riconosciuta ambiguità del titolo).

Uno solo dei rebus spagnoli, in cui l'immagine, invece che disegnata è descritta ("nel gergo dell'enigmistica si tratta di rebus descritti», come spiega Sanguineti stesso), ${ }^{34}$ ha una soluzione immediatamente riconoscibile: GENO-

30. Edoardo SAnguineti, Genova per me, Napoli: Guida, 2004, p. 65. Su Rebus, oltre a Luigi WEBER, Usando gli strumenti di utopia. Tradizione, parodia e riscrittura in Edoardo Sanguineti, Bologna: Gedit, 2004, p. 303-347, cfr. Stefano Agosti, «Sanguineti fuori catalogo», in il verri, n. 44 (ottobre 2010), p. 5-28, ma soprattutto p. 17-28.

31. Lo spunto, il "piccolo fatto» è l'aver pestato il piede ad una visitatrice del Prado, a Madrid («un femminone / tutto DOC, marca Rubens»).

32. Rebus 5 , in Edoardo SANGUineti, Il gatto lupesco, cit., p. 45.

33. "c'è una rivelazione che piacerebbe forse ai venezianisti galizianisti: / sta nascosta in un nunca: dice che già, per me, qualunque ormai si traduce in un mai» (Rebus 4). Cfr. Luigi WEBER, cit., p. 320.

34. Edoardo SANGUINETI, Genova, per me, cit., p. 65. 
VESE GALANTE. E rinvia anche qui ad un'avventura erotica, in questo caso il corteggiamento di una maliziosa «fanciullotta segretaria» d'ambasciata «satanica».

questa frase $(8,7)$ da ventaglio, non firmata, non datata, è un ritaglio banale, da un giornale:

un uomo, che porta un GE sopra una spalla destra, suda, per una sega, seriamente, lì alle prove con una lignea e liscia cosa numero 9: seguono due finestre, con le imposte quasi del tutto aperte, legate con un'L:

(esempio: quando M:M: mi rimbrotta, in ambasciata, la fanciullotta segretaria ispanica, e dice: quella, la devi sculacciarmela:

(e quella è quella che mi spedì, satanica, a succhiarmi un café solo, da solo, in una tasca Losca): io ho aggiunto pronto, allora, con un hola: evviva arriba, mica è un'idea cattiva: che fu un complimento bastante, di strisciante sostanza pensante):

(morale al $124^{\circ} \mathrm{D}$, nella calle del Duque de Medinaceli, è sempre quella, ancora, quella che poi, nell'ora del dipartirsi: ci vuole, disse, e ci sta bene, qui, un addio tanto iberico):

ca, invece, arrivederci, e, senza strazio, a presto), per te (cioè per me):

(che signifi(vedi, al prossimo numero, il risultato arcano di un bacio un po' gitano): ${ }^{35}$

Qui il programma del «piccolo fatto quotidiano» (collocato con la solita minuziosa indicazione della topografia cittadina) realizza anche l'idea della poesia come enigma (o come test) caratteristica di Rebus riproponendo la doppia interpretazione (quella della soluzione del rebus e quella della poesia in quanto testo che deve essere comunque decifrato), che prende spunto da un rebus disegnato, ricavato da un ritaglio di giornale (è Sanguineti stesso a spiegarlo). L'ecfrasi (la descrizione del disegno) si sviluppa nel racconto della propria avventura erotica che coincide con la sua soluzione. La coincidenza fra la soluzione del rebus conservato da tempo, e l'inaspettata occasione offerta dalla situazione vissuta consente una doppia autoironia: sia nel procedere dell' «avventura», attraverso i giochi linguistici ("café solo, da solo», "tasca losca»), ma appunto nell'essere lui, Sanguineti (il genovese galante) l'"esempio" per la soluzione del rebus. E l'autocommento (in Genova per me) a distanza di anni, sarà ancora giocato su questa autorappresentazione («mi metto in scena», «la galanteria disinvolta del galante genovese da me felicemente interpretato»), sottolineando il fatto (lo definisce «un rebussino a puntate») di aver taciuto, creando così un nuovo malizioso enigma, il seguito dell'avventura.

Invece in Rebus 2 la riflessione sullo «sdoppiarsi», che nasce («scopro dovunque») dall'incontro con «i sosia più diversi di smarriti e dispersi», ${ }^{36}$ per le

35. Edoardo SANGUINETI, Il gatto lupesco, cit., p. 47.

36. In una galleria, anche in questo caso, beckettiana di "gente spaventosamente asincronizzata: (e asintopizzata / e asintonizzata): e sformata [...]». 
strade di Valencia "tra il Micalet e il Mercado», provocava un ben diverso turbamento, quasi uno svenimento. E per contrasto nasce il sentimento dell'assenza della moglie («tu non ci sei, tu che mi manchi l'unica:»), ${ }^{37}$ assenza che in Rebus 6 è ribadita dall'uso del pronome personale di seconda persona ( ritorno grondante di arguzie dottorali», "ti ho contemplato un arcobaleno smisurato») nel racconto (è una classica "Postkarte») del viaggio di ritorno da una conferenza a Salamanca.

Questa assenza è collegata ad un'altra dimensione, che si insinua così nella frenesia del viaggio, quella della «bilocazione» (come la chiamerà in Cose 63). Ed è questo, per il Sanguineti viaggiatore, uno dei nodi fondamentali che l'esperienza poetica mette maggiormente in evidenza.

Anche nelle poesie degli anni successivi, quelle appunto di Cose (19962001), che con Rebus ha anche l'analogia del titolo, il viaggio (il primo dei rebus, abbiamo visto, comincia: "qui ogni destinazione è un destino») è la frenetica casualità di «un dappertutto ormai ovunque» (Cose 1 ), riflesso di un mondo globalizzato, ${ }^{38}$ in cui al posto della struttura del viaggio, troviamo l'accostamento di realtà fortemente contrastanti: ad esempio, la riflessione storico-politica innescata dalla visita alla ex-Jugoslavia in fase ormai postbellica (Cose 47), da un lato, e, dall'altro, la privata avventura di una notte madrilena, che provoca il ricordo genovese di una discussione sul tragico (Cose 55).

L'inizio è un trasferimento in taxi, alla Fundación Juan March, per vedere la mostra del pittore espressionista Kart Schmidt-Rottluff facendo tappa al Prado, ma solo per dare un passaggio ad una poetessa romena («ho scortato una Simona poetica (una rumena di Codlea, in taxi, come sempre) / sino all'ingresso del Prado: poi l'ho continuata da solo, la mia corsa [...]). Continua in un ristorante (Casa Manolo alla Princesa): e qui, mentre intorno «la movida» del sabato sera «perdura, dura», l'abbondante bevuta («una bottigliona di Paternina Rioja (banda azul), e dopo un sorbete / de aguardiente de orujo, con pasas») gli fa temere di non riuscire a raggiungere l'albergo («se arrivo al Tirol»), dove poi, in realtà riesce ad arrivare, con la precisa citazione del numero della stanza («ma sono alla 405») e l'inserimento del particolare del rumore proveniente dalla stanza vicina dove «una puttana fa chiasso [...] ha già rotto un bicchiere, si sbatte)», fino al finale epigrammatico: «(vedi ho vissuto per essere un macho: e se non è per questo, perché vivere)». 39

Non semplice aneddoto, ma vera e propria poesia-racconto, dove il montaggio di tipo espressionistico (lo sottolinea Sanguineti nell' autocommento) ${ }^{40}$ lega e collega i frammenti apparentemente senza relazione fra loro e in una casualità temporale. ̇̀ quel montaggio cinematografico — spesso evocato da Curi- che trova qui una definizione precisa nel probabile, anche se non espli-

37. Edoardo SANGUineti, Il gatto lupesco, cit., p. 42.

38. Interessanti al riguardo le osservazioni di Luigi WEBER, op. cit., in particolare alle p. 370-373.

39. Edoardo SANGUINETI, Il gatto lupesco, cit., p. 394.

40. In Edoardo SANGUINETI, Genova, per me, cit., p. 28-40. 
cito, riferimento a Buñuel, quando è precisato come «post-espessionismo di tipo ispanico» ${ }^{41}$ (rimandando così anche alla suggestione del film di Pabst che, secondo la testimonianza di Niva Lorenzini, aveva impressionato Sanguineti al tempo dell' Invenzione di Don Chisciotte). ${ }^{42}$

Come abbiamo visto il viaggio è diventato, da Rebus in poi, uno strumento (un trucco?) per sdoppiarsi, guardarsi da fuori. C'è un verso, in Cose, l'incipit del n. 63, che rende questa idea:

ho praticato le bilocazioni, per riuscirmi a vedermi: fuori di me, fuori di te, in un vuoto: 43

Il viaggio è, dunque, «in un vuoto» (ed è probabilmente per questo che viene colmato dai mille minuti particolari), una di queste «bilocazioni» attraverso cui Sanguineti riesce a vedersi, «fuori di me, fuori di te» e la conferma viene, mi pare, soprattutto dai testi di viaggio in cui è esplicitata l'assenza della moglie, come Corollario 6, prima di atterrare a Madrid (maggio 1992): «se mi strappo da te mi strappo tutto: [...] ritorno in me quando ritorno in te: vivo ancora per te, se vivo ancora:». ${ }^{44}$

Cosicché, tramata di allusioni all'avventura madrilena di Cose 55 (novembre 2000), la nuova poesia dedicata a Madrid (in un viaggio questa volta con la moglie, pochi mesi dopo), Cose 58 (febbraio 2001), può ragionevolmente apparire come una restituzione.

Una restituzione soprattutto erotica ( ma siamo stati, devi ammetterlo pure, mostruosamente felici, / noi due»), immancabilmente narcisistica («io sono stato un re, un mago, credo, spero. Il tuo mago, / il tuo re»), sancita da un lato dalla trionfalmente triviale discesa/ascesa dal putridero e dalla basilica dell'Escorial al Museo Erotico (calle Doctor Cortezo), passando per i ristoranti Oter Epicure (calle Claudio Coello) e Vips (occasioni anch'essi di allusioni erotiche), dall'altro dalla autorappresentazione come don Juan (corretta con un buen Juan) e dalla citazione di tre libri di Bernard Montorgueil. ${ }^{45}$

D'altra parte, dopo l'avventura notturna, in Cose 55, la «bilocazione» aveva prodotto il correre del pensiero al ricordo di una serata genovese, la partecipazione a un dibattito sul tragico, inserito prima dell' "epigramma terminale» (che è ancora riferito alla notte madrilena):

[...] (è soltanto per te che l'altra sera, a Genova, al Teatro della Tosse, ho spiegato (e capito) che cosa che è, davvero, una tragedia):

essere un macho: e, se non è per questo, perché vivere?): 46

(vedi ho vissuto per

41. Si vedano al riguardo, per l'incidenza stilistica, fin da Laborintus, del montaggio cinematografico surrealista, le osservazioni di Erminio Risso, op. cit., p. 34.

42. Niva LorENZINI, Postfazione, cit., p. 160.

43. Edoardo SANGUINETI, Il gatto lupesco, cit., p. 403.

44. Ibidem, p. 264.

45. Ibidem, p. 397.

46. Ibidem, p. 394. 
Commentando la poesia, ${ }^{47}$ Sanguineti ribadirà ancora come l'aporia indissolubile del tragico si sia perduta con l'avvento della società borghese e come sia rimasta solo la possibilità del postespressionismo, quello di cui ha dato un saggio nella parte «spagnola» della poesia.

Introducendo questo commento Sanguineti scrive che quella rievocazione genovese era poco più che un segno (come l'indirizzo apposto a una cartolina) e che "un po" tutti luoghi della sua vita, nei suoi scritti, effimeri e durevoli, sono presenti così, per strappi marginali, per fragili lampi, come le tessere nane di un puzzle molto più largo, come un qualunque frammento di mondo». ${ }^{48}$

Anche per questo, pur avendo indugiato in questa occasione sulle poesie «spagnole» di Sanguineti, sarebbe arbitrario cercare di rintracciare una specifica logica, al di là di quella dell'occasione biografica, sia nelle citazioni (spesso minuziose) di luoghi e particolari «locali», sia nel dialogo che il testo italiano instaura con la lingua spagnola (che è caratteristico del polilinguismo «da viaggio» di Sanguineti ed ha funzione e caratteristiche ben diverse dall'uso, dal prelievo di testi altrui in lingua originale di altri suoi testi).

E per non ingenerare confusione vorrei avvertire, in conclusione, che nel caso di Sanguineti il legame coi luoghi non va sopravvalutato, almeno in partenza e che semmai la Spagna, come qualsiasi altro luogo visitato da Sanguineti è innanzitutto un luogo «allegorico», "realisticamente allegorico».

47. Edoardo SANGUINETI, Genova per me, cit., p. 40-41.

48. Ibidem, p. 37. 